\title{
Acquisition of CD4-Dependence by CD4-Independent SIV Passaged in Human Peripheral Blood Mononuclear Cells
}

Sujatha lyengar ${ }^{1,2}$ and David H Schwartz ${ }^{1,2^{*}}$

\begin{abstract}
Background: Chemokine receptors (CKRs), the primordial receptors for primate lentiviruses, are sufficient to mediate virus-cell fusion. Several different fusogenic CKRs and related receptors provide a broad potential host cell range, presumably advantageous for viral spread within a given infected individual, and across species. By contrast, the additional constraint of obligatory CD4 binding, just prior to CKR engagement, radically restricts potential host cells within an individual (or lymph node microenvironment), and might also limit xenotransmission, as CD4 sequences vary among primates. In spite of these potential drawbacks, CD4 dependent entry for SIV and HIV is the rule rather than the exception, and is generally thought to have evolved by selection for 1) stabilization of virus-cell surface interactions, and 2) conformational shielding of readily neutralized CKR binding epitopes. CD4 binding residues of SIV and HIV envelope are recessed, (relatively hidden from immune detection) and may exhibit a strong degree of automimicry, thus benefitting from self tolerance.

Documented evolution, within individual macaques, of neutralization-resistant CD4-dependent SIV, derived from CD4-independent inocula, supports these ideas, but does not explain CD4's exclusive role as the penultimate receptor-even more striking, given the wide diversity of CKRs and other surface molecules that can serve as actual fusion receptors for SIV. We, therefore, explored the additional, non-exclusive, hypothesis that surface CD4 on leukocytes is a marker of a more favorable host cell environment, as compared to CD8, NK, or B cell surface markers.
\end{abstract}

Results: We demonstrate progressive in vitro evolution of two SIV strains to CD4-dependence (and CXCR4 tropism) in normal human PBMCs (hPBMCs). The two CD4-independent strains of SIV tested developed nearly complete CD4 dependence over several months of serial passage in hPBMCs, correlating with a limited number of nonsynonymous env region mutations, some previously reported to be determinants of CD4-dependency. The initial ability of SIV stocks to grow to significant (albeit, relatively low) levels in CD4(-), CD14(-) cells was also lost with long term passage. Rapid emergence and subsequent prominence of $G \rightarrow A$ and $A \rightarrow G$ mutations within env regions associated with CD4 dependence was seen.

Conclusions: Progressive acquisition of strict CD4 tropism, independent of immunoselection, supports the idea that surface CD4 identifies optimal host cells having intracellular environments most favorable to viral replication. The prominence of mutations involving $G$ to $A$, or $A$ to $G$, suggests that APOBEC 3 mediated infidelity may facilitate rapid switching of cell surface receptor usage within SIV swarms encountering fluctuating availability of optimal $\mathrm{CD}^{+} \mathrm{CKR}^{+}$targets. These observations of non-immune selection are compatible with, and may accelerate, simultaneous selection for previously described CD4-dependent neutralization resistance in vivo.

Keywords: SIV, Tropism, CD4 independent, Chemokine receptor, Selection, Evolution

\footnotetext{
* Correspondence: dschwart@fastmail.fm

'Department of Molecular Microbiology and Immunology, Bloomberg

School of Public Health, Johns Hopkins University, Baltimore, USA

${ }^{2}$ Current address: Jurist Research Department, Hackensack University Medical

Center, 40 Prospect Avenue, Hackensack, NJ 07601, USA
} 


\section{Background}

SIV has crossed many primate species barriers during its evolution to HIV [1,2], and the widely divergent outcomes of infection (from benign subclinical to rapidly pathogenic) reflect multiple host - virus interactions. Among these are coreceptor usage [3,4], and cell subset tropism within progressively colonized microanatomic niches [5-7]. Lin et al. [8] have summarized the evidence that chemokine receptors (CKRs) were the primordial lentivirus receptors, with the evolution of penultimate CD4 binding a more recent development in primate hosts. Most SIV (and essentially all HIV) exhibit strong CD4 dependence, which is generally thought to reflect the selective advantage of high affinity CD4 binding for stabilization of cellvirion interactions, and shielding of chemokine receptor (CKR) binding sites from neutralizing antibodies $[9,10]$.

Yet, with respect to stabilizing membrane interactions, other cognate pairings (e.g., viral membrane ICAM - host membrane LFA-1) stabilize cell-virus coupling during retrovirus docking. Similarly, the explanation of CD4 dependence as a means of protecting readily neutralized fusogenic CKR binding epitopes begs the question: "Why, despite fusogenic receptor diversity for SIV and HIV, has CD4 emerged as the exclusive selecting co-receptor, rather than functionally analogous CD8, or some ubiquitous surface molecule (e.g., CD45)?" Our earlier demonstration that CD4 co-caps with CKRs on the surface of gp120 or virion exposed huPBMCs, and that blocking this capping prevents infection [11] does not, by itself, explain why CD4 should have eclipsed all other molecules in this role, unless it were unique among surface molecules in its actin-mediated association with CKRs. This would appear to be ruled out by work of Tardif and Tremblay $[12,13]$ demonstrating actin-mediated LFA-1 clustering, post-HIV binding, and by observations from several laboratories (e.g., ref [14]) on the co-clustering of LFA-1, CD8, and CKRs at the immunological synapse of CTLs.

CD4-independent SIV and HIV-2 strains can be selected in vitro $[1,8,9,15,16]$, and isolated from the CNS of macaques $[17,18]$ and blood of rapid progressor (RP) or late stage $[10,19]$ animals - settings with a paucity of CD4+ targets. Similarly, CD4 independent HIV-1 has been recovered from the CNS of HIV + individuals and (very rarely) AIDS patients with extreme CD4+ cell depletion, in some cases from CD8+ cells [20,21]. Of note, Vodros et al., [3] demonstrated, within 12 days of infection, increased heterogeneity of circulating SIV envelopes capable of mediating CD4-independent fusion in vitro. They suggested that local depletion of CD4+ T cells available, within initially infected sites during the acute phase, might select for $\mathrm{CD} 4$ independence.

Replacement of injected CD4-independent RP SIV by CD4-dependent virus in macaques has been attributed to neutralizing antibodies (Abs) arising roughly 40 weeks post infection, while (rare) persistence of CD4-independent SIV in RP animals was interpreted as the consequence of feeble, ineffective, neutralizing Ab responses [10,19,22]. But RP macaques also have rapid loss of almost all susceptible CD4+ lymphocytes, so, likewise, non-immune selective pressures favoring $\mathrm{CD} 4$ tropism would be absent, allowing persistent CD4-independence at the level of non-immune selection, as well. Decreased fitness in macaque PBMCs of CD4 independent SIV from rapid progressors (vs. parental CD4-dependent virus) was considered in vitro artifact $[10,22]$. However, rapid progressor (RP) SIV was not examined for in vitro evolution to parental CD4 dependent phenotype and/or genotype during maintenance in vitro. Referring to data not shown, Vodros et al.[3] noted that "extensive passage on hPBMCs" of a largely CD4-independent SIV strain "resulted in inoculum from which strictly CD4-dependent clones were derived, suggesting that CD4-dependent viruses may be more fit under these conditions."

One previously unexplored potential pressure for the selection of CD4 as the exclusive co-receptor for HIV is that CD4 cells are intrinsically better internal hosts for SIV (and HIV) replication than other primary cells, for reasons (e.g., cell-type specific restriction factors) unrelated to surface CD4. This hypothesis predicts that, among a swarm of SIV, CD4 dependent phenotypes will out-compete strains with different (e.g. CD8 co-receptor) or more promiscuous (e.g., CKR only) entry criteria, because they selectively infect host cells that are intrinsically more productive. This advantage should exist in the absence of specific anti-SIV humoral or cell mediated immunity, as long as there are sizable proportions of both $\mathrm{CD} 4+$ and CD4(-) targets with suitable surface CKRs for binding and fusion. Selection for CD4 dependence might diminish in the face of drastically reduced availability of activated CD4+ cells (as in RP macaques, or macaque CNS infection, or within a "burned out" node, depleted of in situ CD4+ activated T cells), or during xenotransmission to a primate whose CD4 had much lower avidity for envelope.

We set out to test the scenario of selection for CD4 tropism in the absence of immune pressure, by long term culturing in normal hPBMCs of two SIV strains which exhibit CD4 independence during initial rounds of infection in hPBMCs [23,24].

\section{Results}

SIV stock strains are CD4 independent in hPBMCs, and productively infect hPBMCs depleted of CD4+ and CD14+ cells

SIV mac239 ${ }_{\text {CEMx174 }}$ and 17E-Fr stocks, both produced in CEMx174 cultures, were inoculated into wells containing PHA stimulated hPBMCs. After continuous passage of SIV mac239 in CEM x 174 cells, the resulting 
stock, which we here call SIV mac239 ${ }_{\text {CEMx174, is CD4 }}$ independent for infection of human PBMCs, while remaining CD4 dependent in macaque PBMCs, as previously reported $[23,24]$. This fortuitous observation gave us a second SIV strain, along with SIV 17E-Fr (which was genetically unchanged after passage in CEMx174 cells) to analyze for progressive acquisition of CD4 dependence in hPBMCs. This phenotype was not due to surface components incorporated from the CEMx174 line, in which case CD4 independence should have been lost after just one round of replication in hPBMCs. Rather, sequencing of stock SIV mac239 ${ }_{\text {CEMx174 }}$ indicates the presence of several mutations differing from the parental SIV mac239 [GenBank accession number M33262], and previously associated with CD4 independence (discussed below).

Parallel cultures of equal numbers of anti-CD4 treated hPBMCs, or PBMCs immunodepleted of CD4+ and CD14+ cells, were established at the same time. Separate experiments demonstrated comparable levels of day 5 proliferation, as measured by thymidine incorporation, among these various cell populations (data not shown). As shown in Figure 1A, anti-CD4 mAb treated hPBMCs supported high replication $(>8,000 \mathrm{pg} / \mathrm{ml} \mathrm{p} 27)$ of both initial stock strains of SIV (off-scale at 1:10 dilution), as did untreated PBMCs. The Figure 1A supernatants were not diluted to endpoint, but in similar experiments using the stock viruses, anti-CD4 mAb treated cultures supported several fold higher levels of p27 production than untreated control cultures, as determined by endpoint dilution of day 7 or day 10 supernatants (Figures 2 and 3, discussed below).

Lack of surface CD4 dependence for entry does not formally prove that cells other than CD4+ lymphocytes are actually being infected. CD4+ cells might still be selectively targeted, via some non-CD4 mediated mechanism. This point was addressed by documenting moderate growth in hPBMCs rigorously depleted of CD4+ lymphocytes and CD14+ monocytes $(<1 \% \mathrm{CD} 4+,<1 \% \mathrm{CD} 14+$, as determined by flow cytometric analysis), albeit with significantly lower levels of virus production $(\sim 700$ and $1100 \mathrm{pg} / \mathrm{ml}$ p27, respectively, of SIV mac239 ${ }_{\text {CEMx174 }}$ and 17E-Fr, Figure 1A). The decreased growth of SIV in CD8 $+\mathrm{T}$ cells, B cells, and other remaining CD4(-) CD14(-) mononuclear cells, compared to anti-CD4 treated whole PBMCs, suggests that, despite blocking and/or downregulation of surface CD4, the CD4+ cells are still being infected, and are producing more virus than the CD4(-) cells from the same source. This is consistent with our hypothesis of a selective growth advantage for virions

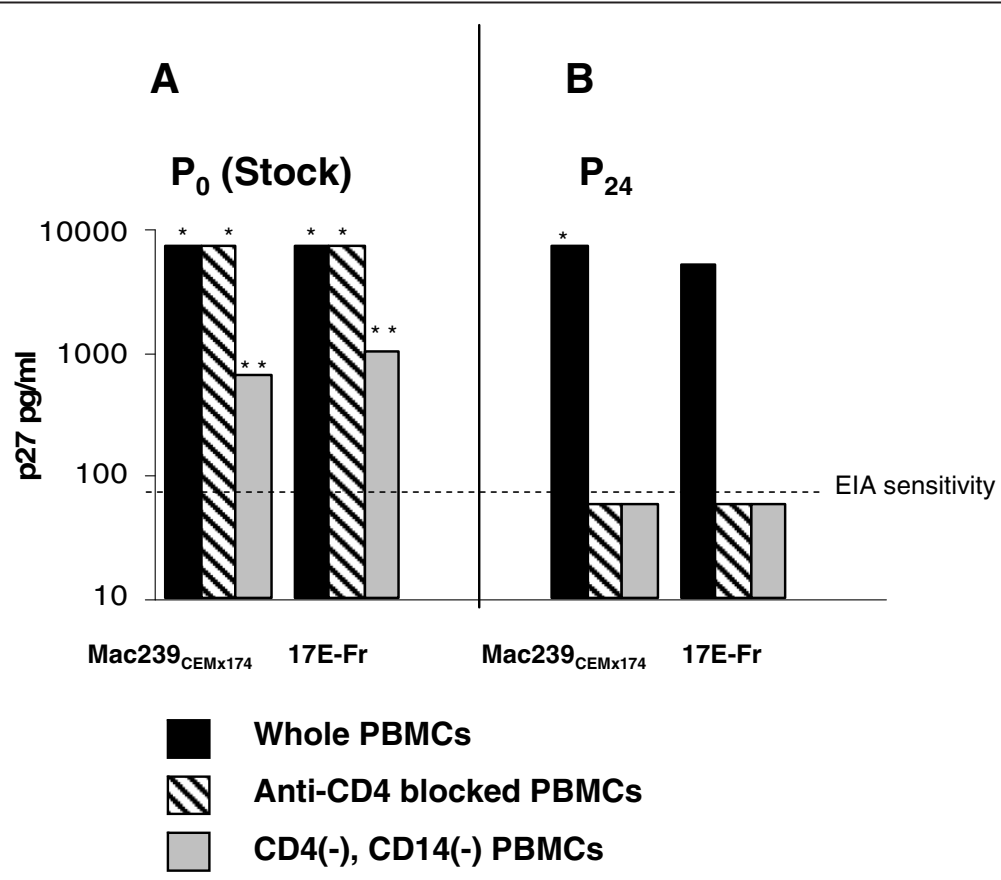

Figure 1 Stock vs. Passage 24 SIV CD4 dependence and infection of CD4+ and CD14+ depleted hPBMCs. A. Day 7 growth of stock SIV mac239 CEM×174 and SIV 17E-Fr in human PBMCs was robust (>8,000 pg/ml p27) whether or not cells were pretreated with CD4 blocking concentrations of Leu3a. Limited supernatant volumes prevented endpoint dilutions, but contained p27 levels that remained off-scale $\left(^{*}\right)$ at 1:10 dilution. ImmunodepletedhPBMCs containing fewer than 1\% CD4+ or CD14+ cells still supported readily detectable levels of viral replication, but at significantly lower levels (** $p<0.0001$ vs. $8,000 \mathrm{pg} / \mathrm{ml}$ ). B. By the $24^{\text {th }}$ passage in allogeneic $\mathrm{hPBMCs}$, both SIV mac239 $9_{\mathrm{CEM} \times 174}$ and SIV $17 \mathrm{E}-\mathrm{Fr}$ had lost the ability to infect PBMCs pretreated with anti-CD4. Moreover, no growth of either strain was detectable above EIA sensitivity (dashed line) in day 7 supernatants of hPBMC depleted of CD4+ and CD14+ cells. 
A Acquisition of SIV CD4 Dependence in Human PBMCs

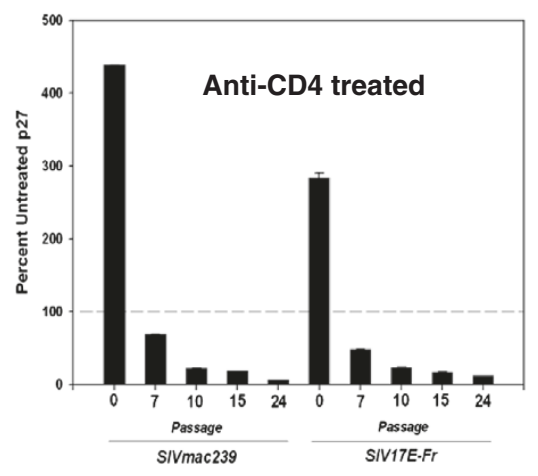

$\boldsymbol{B}$

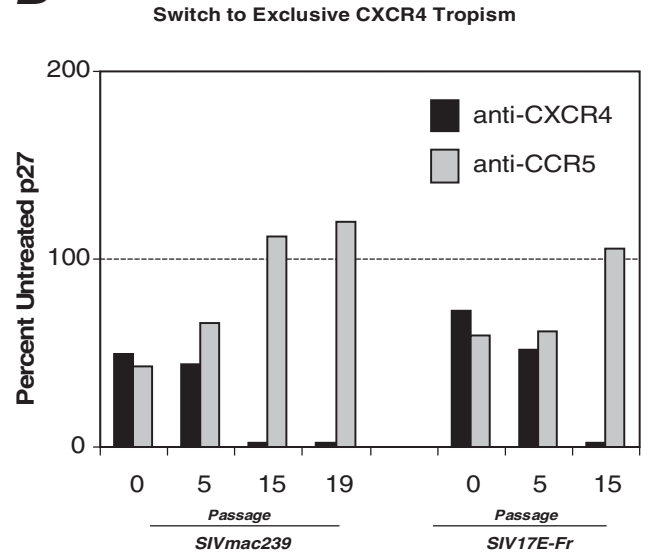

Figure 2 Progressive changes in CD4 and CKR usage by SIV serially passaged in hPBMCs. A. Day 7 supernatant p27 concentrations of hPBMC cultures, transiently treated with Leu3a anti-CD4 mAb during infection, were normalized to simultaneously mock-treated control cultures (100\%, dashed line -- - - ). Initial 4- and 3-fold greater replication in Leu3a anti-CD4 mAb treated cultures of, respectively, SIV mac239 CEMx174 (abbreviated SIV mac239) and SIV 17E-Fr was followed by progressively increasing CD4 dependence during serial passage in unrelated human donor PBMCs. B. By the $15^{\text {th }}$ passage, when both SIV strains had become almost completely CD4 dependent (see $\mathbf{A}$ ), CKR usage in both strains had switched from roughly equal CXCR4 and CCR5 to nearly exclusive CXCR4 tropism, demonstrated by relative reduction of virus growth in cells pretreated with blocking mAbs against CXCR4 vs. CCR5.

entering CD4+ lymphocytes when they are available, even if surface CD4 is not utilized for entry. This level of replication was absent in identically depleted cultures of late passage, CD4 dependent, SIV, which produced only uninfected control background levels of p27 (Figure 1B).

\section{CD4 independent SIV passaged repeatedly in hPBMCs} becomes CD4 dependent in hPBMCs, exclusively CXCR4 tropic, and no longer infects hPBMCs depleted of CD4+ and CD14+ cells

The cultures established in hPBMCs (described above) were re-fed weekly with fresh allogeneic PHA stimulated normal hPBMCs and complete media. Table 1 shows that significant percentages of the non-adherent CD4(-), as well as CD4+ cells present in these cultures at days 1 , 4 , and 7 were positive for either CXCR4 or CCR5 (or both). Moreover, the increasing ratio of CXCR4 : CCR5 expression during the 7 day culture period was not markedly different for CD4+ vs. CD4(-) populations.

Aliquots of supernatant from SIV stocks $\left(\sim 10^{3} \mathrm{TCID}_{50}\right)$ or progressive weekly passages $(\mathrm{P} \#)$ were sampled at $1-2$ month intervals for sequencing, and used to infect normal PHA stimulated hPBMCs, with or without preblocking by anti-CD4 mAb (samples from P0, P7, P15, and P24), or anti-CKR mAb (samples from P0, P5, P13, and P19), or pre-culture immunodepletion of $\mathrm{CD} 4+$, CD14+ cells (P0 and P24). Additionally, P0 and P19 virus samples were pre-incubated with soluble recombinant CD4-IgG (sCD4) prior to inoculating PBMCs.

Surprisingly, initial stocks of SIV mac239 ${ }_{\text {CEMx174 }}$ and SIV 17E-Fr both actually grew better in hPBMCs transiently blocked with Leu3a mAb during initial exposure, than in unblocked controls. Nevertheless, during succeeding passages, CD4 independence was progressively
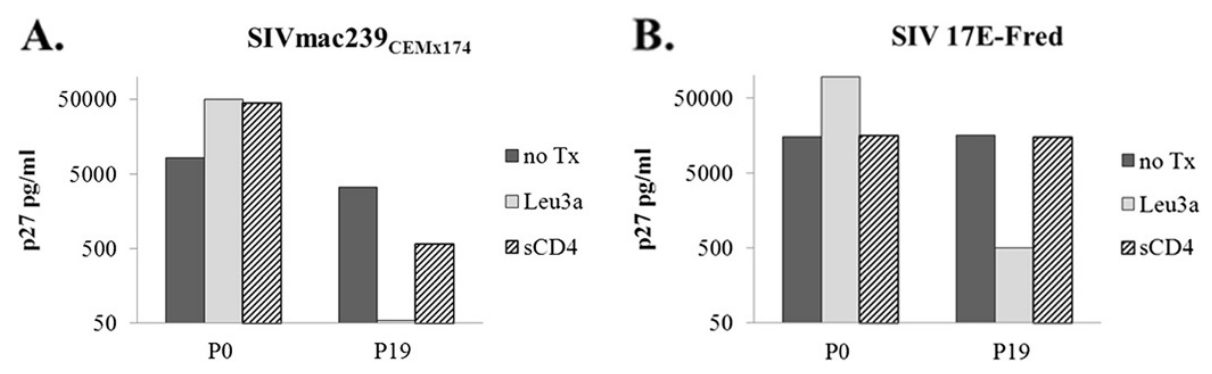

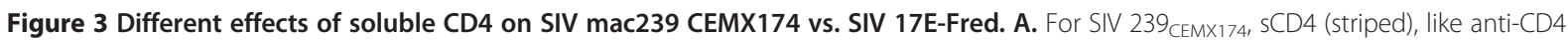
(Leu3a, gray), enhanced infectivity of stock virus, but decreased growth of late passage virus, although to a lesser degree than Leu3a. B. For SIV 17E-Fred, SCD4 appeared to have no effect on stock or P19 virus infectivity. (Note that P0 p27 values reflect day 10 culture supernatants vs. day 7 for P19). 
Table 1 Percentage of CD4+ and CD4(-) non-adherent hPBMCs expressing receptors for SIV during one week cultures

\begin{tabular}{ccccc}
\hline & \multicolumn{4}{c}{ Percentage of Cells Expressing Surface Markers } \\
\hline Surface markers: & CD4(-)CXCR4+ & CD4(-)CCR5+ & CD4+ CXCR4+ & 47 \\
Day 1 & 37 & 22 & 68 & 8 \\
Day 4 & 25 & 17 & 36 & 14 \\
Day 7 & 53 & 7 & 4 \\
\hline
\end{tabular}

Whole human PBMCs were stimulated with PHA + IL-2, and maintained in complete medium (10\% FCS) for one week, with sampling of resuspended cells on days 1, 4, and 7. Cells were dual stained with CD4 and CXCR4 or CCR5 specific mAbs, to determine the percentages of CD4+ and CD4(-) cells expressing CXCR4 or CCR5 at various times. Because co-expression of CXCR4 and CCR5 on either CD4+ or CD4(-) cells was not determined, total percentages of CKR + cells are not calculated by summing the four populations for each day.

lost (Figure 2A), roughly in parallel with increasing CXCR4 tropism (Figure 2B). Additionally, by passage 24, both SIV strains had lost the ability to infect CD4(-), CD14(-) huPBMCs (see Figure 1B).

SIV stock strains are not rendered less infectious by preincubation with soluble IgG-CD4, and evolve discordant sensitivities during hPBMC passage

As shown in Figure 3, results after pre-incubating SIV with soluble IgG-CD4 were somewhat discordant for the two viruses. For P0 SIV 239 CEMX174, sCD4 strongly enhanced infection (as did the CD4 blocking mAbLeu 3a). For P19 SIV 239 CEMX174 isolates, which were almost totally blocked by Leu3a, sCD4 suppressed growth by $>80 \%$. Thus, with respect to SIV 239 CEMX174, sCD 4 pre-incubation reduced infectivity generally comparable to anti-CD4 mAb. However, in the case of SIV 17E Fred, pre-incubation with sCD4 had no effect on CD4 independent infectivity of stock strains or CD4-dependent P19 isolates. Possible reasons for these observed differences are considered in the Discussion section.

\section{Progressive emergence of variants with specific mutations in the envelope region}

We next asked whether there were any common mutations emerging in the strains of SIV that had evolved CD4 dependence (which can be conferred by single mutations $[25,26])$ and, if so, how quickly they emerged. We sequenced both strands of full length envelope from 15 clones, each, of stock SIV 17E-Fr and stock SIV mac239 CEMx174 carried in CEMx174 cells, 15 clones derived from hPBMC passage 7 , and 15 clones from passage 24. Initial SIV mac239 CEMx174 virus, as well as the neurotropic variant SIV 17E-Fr, contained residues (67 Met and 176 Asn) previously associated with CCR5 tropic, CD4 independent (in macaque cells) SIV recovered from brains of macaques initially infected with WT SIV mac239 [27].

Interestingly, both stock virus strains grown in CEMx174 also retained certain key residues previously associated with CD4 dependent infection in non-human primates, including Met at position 165, Ile at 324, and (in 17E-Fred only) Lys at 573,the CD4 contact point of the conserved C3 loop GGDPE region [16-18]. Thus, in accord with most published sequences of CD4-independent SIV grown in macaque PBMCs, our stock virus sequences suggest stably exposed CKR binding sites as the basis for initial CD4 independence in hPBMCs, rather than complete loss of CD4 affinity.

Overall, the 15 clones of envelope from SIV 17E-Fr stock virus were $>99.9 \%$ homologous at the amino acids (aa) level with eachother and the published sequence of this strain [28]. Despite the observed progressive acquisition of CD4-dependence, stock virus and all subsequently passaged 17E-Fr carried Gly at aa 751, a residue previously shown to contribute to CD4 independence in macaque astrocyte-tropic isolates [15]. For SIV mac239 CEMx174, all 15 stock clones were, again, remarkably homogeneous, with $>99.9 \%$ aa homology among the 15 clones overall, and only two clones having more than a single aa differing from this consensus sequence. Importantly, none of this very limited heterogeneity involved aa positions that subsequently underwent mutation during passage in hPBMCs. Figure 4 aligns regions of the starting nucleotide sequences of the two stock strains (P0) with the predominant mutated sequences, for the respective strains, recovered at P24.

For $17 \mathrm{E}-\mathrm{Fr}$, by passage 24 , mutations in nucleotide position 1148 ( $\mathrm{G}$ to $\mathrm{A}$ ) and 2222 ( $\mathrm{C}$ to $\mathrm{T}$ ) were present in all 15 clones sequenced, leading to aa acid changes of Gly to Glu, and Pro to Leu, at aa residues 383 (C3 domain), and 741 (proximal to the transmembrane domain), respectively (Figure 4A). An additional mutation at nucleotide position 2326 ( $\mathrm{C}$ to $\mathrm{A}$ ) was seen in 12 of 15 clones from passage 24, yielding a Leu to Met conversion at aa 776. Of these most common mutations, only $\mathrm{G}$ to $\mathrm{A}$ at nucleotide position 1148 had appeared in 3 of 15 clones by passage 7 , suggesting it may have been critical for the step-wise conversion to CD4-dependent phenotype. Glu 813 Arg was present in 8 of 15 passage 24 clones, and several other substitutions were present at frequencies of $4 / 15$ or less.

The pattern of passage acquired mutations for SIV mac239 CEMx174, was more complicated than for 17E-Fr, with only a single mutation seen for the first time at passage 24 , and not in stock or passage 7 samples 


\section{A.SIV 17E-Fred amino acid substitutions predominant in P24 Clones}

\begin{tabular}{|c|c|c|c|}
\hline Passage & $\underline{\text { region }}$ & & frequency \\
\hline & & 383 & \\
\hline P0 & $\mathrm{C} 3$ & NTDKINLTAPRGGDPEVTFMW & \\
\hline P24 & & NTDKINLTAPREGDPEVTFMW & $15 / 15$ \\
\hline & & 730 & \\
\hline P0 & TM & SYFQQTHIQQDPALPTREGKE & \\
\hline P24 & & SYFQQTHIQQDLALPTREGKE & $15 / 15$ \\
\hline & & 766 & \\
\hline P0 & TM & IEYIHFLIRQLIRLLTWLFSN & \\
\hline P24 & & IEYIHFLIRQMIRLLTWLFSN & $12 / 15$ \\
\hline
\end{tabular}

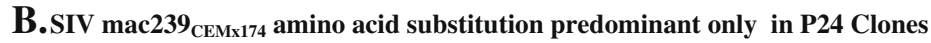

Passage region

$\begin{array}{lllr} & & 180 & 190 \\ \text { P0 } & \text { V2 } & \text { KKEYNETWYSADLVCEQGNNT } \\ \text { P24 } & & \text { KKEYNETWYSVDLVCEQGNNT }\end{array}$ frequency

$14 / 15$

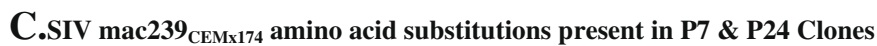

\section{$\underline{\text { Passage }} \underline{\text { region }}$}

P0
P7
P24

P7

P7
P24

P0
P7
P24

P0
P7
P24

P0

P7
P24

P0

P7
P24

P0

P7
P24

P0

$\mathrm{P} 7$
$\mathrm{P} 24$

P0
P7
P24

P0

P24

P0
P7

P7
P24

P0
P7
P24

\section{C1}

$60 \quad 67$

requency

80

DNGDYSEMALNVTESFDAWNN DNGDYSEVALNVTESFDAWNN

V1 $141 \quad 158$

ARVDMVNETSSCIAQDNCIGL

AKVDMVNETSSCIAQDNCTGL
AKVDMVNETSSCIAQDNCTGL

$170 \quad 176$

189

FNMTGLNRDKKKEYNETWYS FNMTGLKRDKKKEYNETWYS FNMTGLKRDKKKEYNETWYS

$210 \quad 217 \quad 230$

C2 HCNTSVIKESCDKHYWDAIRF HCNTSVIQESCDKHYWDAIR HCNTSVIQESCDKHYWDAIRF

$\begin{array}{rlrr} & 300 & 310 & 320 \\ \text { V3 } & \text { ISLNKYYNLTIKCRRPGNKTV }\end{array}$

ISLNKYYNLTMKCRRPGNKTV

ISLNKYYNLTMKCRRPGNKTV

$\begin{array}{llll}330 & 335 & 341 & 350\end{array}$

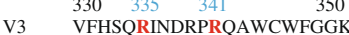

VFHSQPIND

VFHSQPINDRPKQAWCWFGGK

VFHQP

$\begin{array}{llr} & 380 & 383 \\ \text { C3 } & \text { DGS---RRRSGSYLHVDKLQRRV }\end{array}$

DGSWRRRSGSYLHVDKLQRRV DGSWRRRSGSYLHVDKLQRRV

$\begin{array}{llr} & 430 \quad 436 & 450 \\ \text { C4 } & \text { LRAMSYQTNNQHLA-SRQKCL }\end{array}$

LRAMSYQTNNQHLA-SRQKCL

LRAMSY--TNNQHLA-SRQKCL

600

$\begin{array}{lrr}600 & 609 & 620 \\ \text { VLKGPGAAEYLGMCV-TSLPH }\end{array}$

VLKGPGAAEYLGMCV-TSLPH VLKGPGAAECLGMCV-TSLPH VLKGPGAAECLGMCV-TSLPH

$620 \quad 631 \quad 640$

TM $\quad 620$

640
631 HYCTMAXMQV.HQSGTMRLGK HYCTMAXMQV HQSGTMRLGK

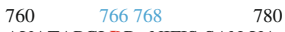

TM AVATAPGLDR--NIFIS-SAN-YA

AVATAPGLGRYNIFIS-SAN-YA

$\begin{array}{lrr}830 & 838 & 850\end{array}$

TM AISMRRSRSSGDLRQRLLRAR AISMRRSRPSGDLRQRLLRAR AISMRRSRPSGDLRQRLLRAR
$14 / 15$

$15 / 15$

$11 / 15$

766: $15 / 15$

$15 / 15$
$15 / 15$

$15 / 15$

$15 / 15$

$15 / 15$

$15 / 15$
$15 / 15$

$15 / 15$
$15 / 15$

$15 / 15$

$15 / 15$

$15 / 15$

$5 / 15$

766: $15 / 15, \quad 768: 11 / 15$

$15 / 15$

Figure 4 (See legend on next page.) 
(See figure on previous page.)

Figure 4 Predominant changes in amino acids between P0 and P7, or P24. Single letter sequences are shown for stock (P0), passage 7 (P7) and/or passage 24 (P24) SIV clones, with the position of each change indicated in blue numbers above the PO sequence, the PO residue in red, altered residues in green, and the clonal frequency of mutations indicated for passages. The variable (V), conserved (C), or transmembrane (TM) regions in which mutations occur are indicated. SIV 17E-Fred (A) required few mutations to become CD4 dependent. By contrast, SIV 239 ${ }_{\text {CEM } 174}$ required multiple mutations by P24 (B) to become CD4 dependent, with many appearing by P7 and persisting through P24 (C).

(Figure 4B). This was a $\mathrm{C}$ to $\mathrm{T}$ mutation in $14 / 15$ clones at nucleotide position 569, yielding Ala to Val at aa 190. Unlike the SIV 17E-Fr findings, several additional nonsynonymous mutations were found to arise relatively early (during or before passage 7) and persist through passage 24, suggesting these changes also contributed to early partial CD4 dependence and increased fitness in vitro. The most consistent changes, seen in all fifteen of the passage 7 , and all fifteen of the passage 24 clones, were: Met 67 Val, Arg 141 Lys, Ile 158 Thr, Asn 176 Lys, Lys 217 Gln, Arg 335 Pro, Arg 341 Lys, 383 Trpinsertion, Tyr 631 His, Asp 766 Gly, Ser 838 Pro. Interestingly, three of these changes were $G$ to $A$ and five were A to G. Notably, almost every mutation emerging during early (P7) hPBMC passage of SIVmac239 ${ }_{\text {CEMx174 }}$ and persisting through $\mathrm{P} 24$, resulted in the restoration of an aa present in the canonical SIVmac239 sequence. Additionally, Ile 310 Met, and Tyr 609 Cys mutations were seen in all 15 SIV mac239 CEMx174 clones at passage 7 (each reflecting an A to $G$ mutation), but only in 11 of 15 clones at passage 24 , with the other clones containing the original stock virus residues. This could have been due either to expansion (i.e., reemergence) of clones carrying the retained stock aa residues, or reverse mutation back to initial sequences (via G to A mutation). As mentioned above, in every case, the emergent dominant mutations present in P7 and P24 clones returned the sequence to the original published SIV mac239 residue, so that P24 SIV mac239 CEMx174 was almost identical to the canonical SIV mac239.

\section{Discussion}

An ability to replicate in the setting of severely limited CD4+ targets (e.g., advanced disease or CNS infection) would be a selective advantage for SIV or HIV at various times in the course of a typical infection. CD4 independence might also facilitate xenotransmission to primate species exhibiting dramatically lower binding constants for viral envelope interaction with CD4 [29]. How often this has occurred and to what extent species-specific CD4 has been a barrier to xenotransmission remain unknown. Despite the apparent advantages of CD4 independence, it is the exception rather than the rule, suggesting a strong selective advantage for CD4 dependent viruses.

Reacquisition of CD4-dependence has been observed for neurotropic CD4-independent SIV injected peripherally into monkeys, and has been correlated with, and attributed to, development of neurotropic strain specific neutralizing Abs. Here, we have presented the first evidence that progressive reacquisition of CD4 dependence can evolve in human cell cultures of SIV, over a similar period of time, entirely independent of specific immunity. These observations in no way contradict the importance of immune selection for CD4 dependence in vivo, but do suggest other components, such as cell type specific restriction factors, may exert selective pressure.

The possibility that rare residual CD4+ cells in CD4(-) CD14(-) hPBMCs might be the source of detected initial SIV strain replication is exceedingly remote, as $<1 \%$ CD $4+$ cells scattered among non-infectable neighbors would be unable to support the efficient cell-to-cell transmission that accounts for $>95 \%$ of in vitro replication [30]. Also, CD4+ cell contamination cannot explain why viral replication failed to reach detectable levels in identically depleted cultures of late passage, CD4 dependent, SIV.

Figure 5 models a 9-fold selective advantage that would be manifest after only three rounds of replication for two hypothetical strains of virus-one CD4 independent and capable of promiscuous entry into any PBMC expressing appropriate surface CKR, the other restricted to $C D 4+$ cells bearing suitable surface CKR. The model assumes identical efficiencies of entry for all permissive combinations of virion and host cell, and makes the further assumptions that CD4(-) and CD4+ lymphocyte targets are available at a ratio of roughly 2 : 1 , that encounters with targets are stochastically random, and that the reproductive advantage of entering CD4+ vs. CD4(-) cells is just 3-fold. In reality, the reproductive advantage appears to be one or two orders of magnitude greater, and hundreds of replication cycles typically occur prior to detection of seropositivity. Under such conditions, the chance of isolating CD4 independent strains from the peripheral circulation is small.

Up to four-fold increased infection of hPBMCs by stock SIV in the presence of anti-CD4 mAb, seen in several experiments (e.g., Figures 2 and 3), was probably due to more efficient envelope binding of partially capped, actin associated CKRs following Leu3a crosslinking of CD4 [11]. Despite any such enhanced CKR cell surface topography that might facilitate CD4independent viral infection of transiently Leu3a treated cells, CD4 dependence increased progressively with successive passages, and became nearly absolute by P24. 


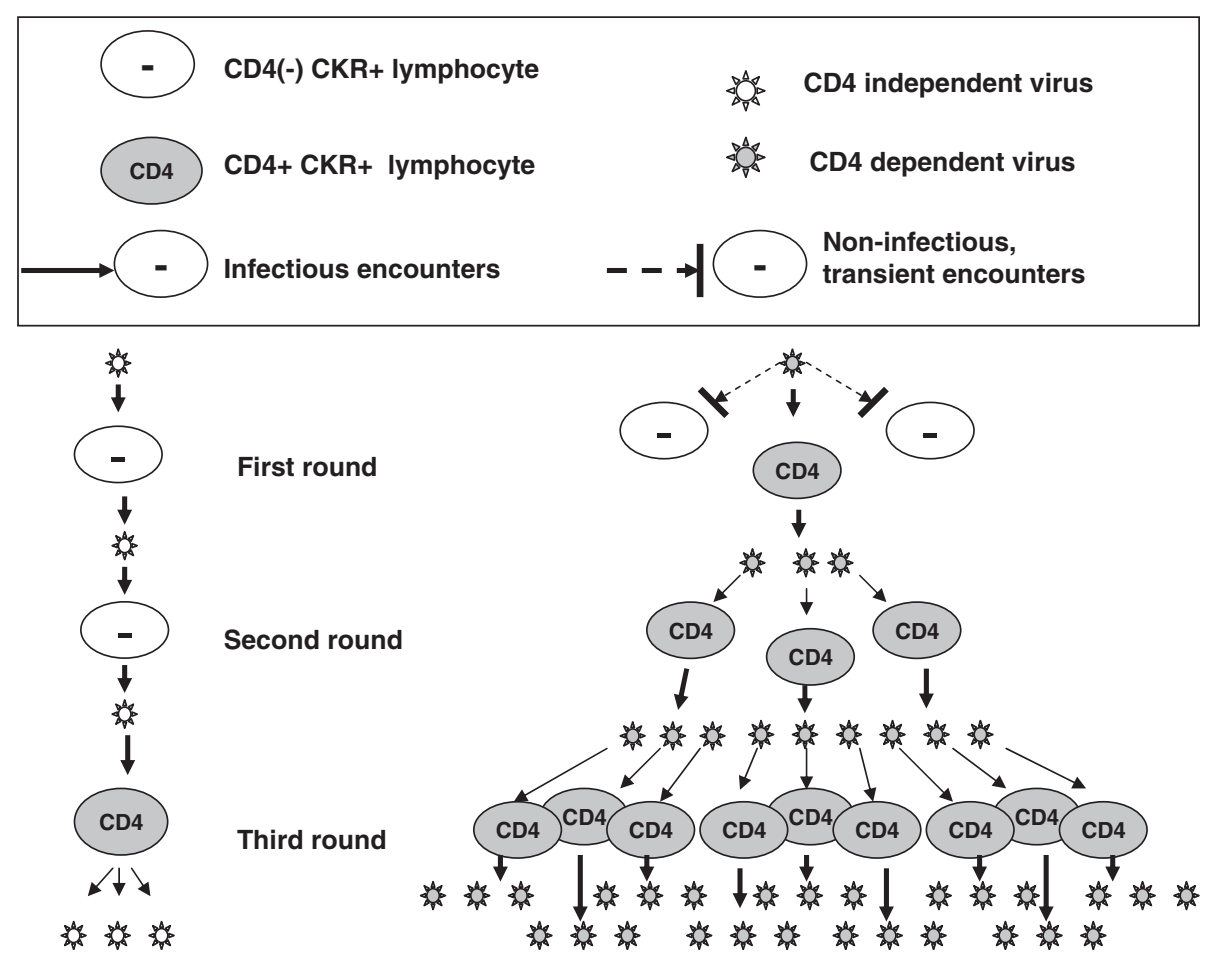

Figure 5 Modeling the selective advantage of strict CD4 dependence vs. promiscuous entry. The number of CD4 dependent (shaded) : CD4 independent (unshaded) virions after 3 rounds of replication is 27:3. This illustrative hypothetical example makes the following simplifying assumptions: $\mathrm{CD} 4(-)$ and $\mathrm{CD} 4+$ potential host cells in a sufficiently activated state, and bearing the appropriate CKRs, are abundantly present in a ratio of $2: 1$, yielding an encounter with CD4+ targets once in every three virus-cell interactions, on average (transient encounters of CD4 dependent virus with CD4(-) cells are shown only for the first round); the relative level of infectious virus released per infected CD4+ cell is $3 x$ that of an infected CD4(-) cell, and is the same for either strain of virus; the three types of permissive encounters between viruses and host cells have equal efficiencies of entry.

The reasons for strain differences in the results with soluble Ig-CD4 are not readily apparent, but could relate to one or both of the two established mechanisms for sCD4 mediated inhibition of HIV: steric blocking of cellular CD4 ligation, and induced envelope shedding from virions [31]. In addition, the two strains of initially CD4independent SIV might show different initial CD4 binding affinities, and different degrees of change in affinities during passage. Indeed, some envelopes have been found not to bind multimeric sCD4 at all, perhaps due to tight trimer packing, while certain glycosylation sites have been found to confer relative resistance to $\mathrm{SCD} 4$ inhibition [32]. Moreover, effects of sCD4 can include concentration dependent enhancement of infectivity, which may vary with the presence or absence of surface CD4 expression of target cells [31,33-35]. Mono- and multimeric sCD4 were previously shown to enhance SIV mac239 infectivity on CD4+ and CD4(-) CCR5+ cells [31,33-35], presumably by stabilizing CKR binding sites that are already partially exposed, and we suspect that this mechanism is operative for our stock SIV mac239 CEMx174 as well. By passage 19, this enhancement was replaced by predominant inhibition. The Leu3a blocking data support the interpretation that exposure of cryptic CKR binding sites in late passage isolates requires prior engagement of CD4. In this context, either shedding of envelope, or steric interference with envelope-CKR docking, could account for the suppressive effect of sCD4.

In contrast, the relative lack of effect of sCD4 coincubations on either initial or late passage SIV 17EFred suggests that changes in CD4 binding affinity were not involved in the acquisition of CD4 dependence. Rather, new conformational masking of CKR binding sites may have evolved in the late-passage isolates, requiring relatively prolonged contact with surface CD4 to allow fusogenic binding. This observation is consistent with the minimal binding of envelope to soluble CD4, as has been previously described for some isolates, or binding with only subtle changes in SIV env conformation, as seen in recent cryo-electron studies [36]. Thus, mechanistic interpretation of discordant $\mathrm{sCD} 4$ effects is not straightforward, but these experiments underscore the fact that the structural/functional differences accumulating between initial and late passage SIV mac239 CEMx74 viruses were somewhat different in kind from those accumulating in SIV-17E-Fred. 
The observed switch from mixed CCR5 and CXCR4 usage in hPBMCs to predominantly CXCR4 usage by both strains has been described for SIV cultured in hPBMCs [3], and may be functionally related to acquisition of CD4-dependence [25]. Lauren et al. [37] have suggested the two are interrelated properties for SIVsm, but this is not an absolute requirement. Which of the envelope mutations associated in our experiments with CD4 dependence determine CKR tropism is undefined. The exact changes at aa positions 47 in the $\mathrm{C} 1$ domain, and aa 316, 324, and 328 in the V3 loop, described by Del Prete et al. [38] as conferring CXCR4 dependence on SIV mac239 were not seen. However, mutations did occur at nearby positions within the same regions (aa positions $67,334,340$ ), and may have played a similar role in the context of the other mutations arising in SIVmac239 CEMx174 as it developed increasing CD4 dependence.

PHA/IL-2 stimulated PBMCs contain more CXCR4+ than CCR5+ expressing cells [39], and this difference is more pronounced in CD4(+) vs. CD4(-) populations in culture [Table 1], but relative abundance of CXCR4 vs. CCR5 cannot be the sole reason for the tropism switch, as both R5 tropic stock strains were grown in the CEMx174 line that express $\sim 20,000$ CXCR4 surface receptors per cell and no detectable surface CCR5. In vivo switching of CCR5 tropic to CXCR4 tropic SHIV has also recently been described in macaques, and is correlated with enhanced, rather than de novo, binding to $\mathrm{CD} 4$, based on a more open molecular conformation that may facilitate heterotypic binding to CKRs [40].

The early appearance of $\mathrm{G}$ to A mutations in both SIV mac239 ${ }_{\text {CEMx174 }}$ and 17E-Fr, along with the apparent back and forth mutations between $G$ and A seen with SIV mac239 CEMx174 in the course of evolving binding requirements, suggests a role for APOBEC 3 mediated $G$ to A mutations in rapidly generating optimal viral fitness for growth in hPBMCs. This could help account for why primate retroviruses have evolved only partial inhibition of APOBEC 3 activity. It is interesting to note that, in all but one instance, mutations acquired by SIVmac239CEMx174 restored the sequence to that of the canonical SIV mac239.

\section{Conclusions}

Our findings suggest that in the absence of HIV specific immunity, there are still strong selective pressures favoring replication of SIV clones that limit their entry to CD4+ cells. Seen in this light, surface CD4 becomes an important "Welcome" sign for primate retroviruses, signaling the most supportive host cells, rather than merely an opportune foothold by which to gain entry. The unique intracellular features of CD4+ T lymphocytes, compared with minimally productive CD8+ T cells, NK cells, or B lymphocytes, accounting for such strong selective pressure in vitro and in vivo remain unknown, but may hold the key to future clinical interventions. Recent studies have identified restriction factors for DCs $[41,42]$, and it is possible that these or other intracellular factors limiting primate lentivirus replication will be found in CD4(-) lymphocytes, as well.

\section{Methods}

\section{Cells and reagents}

The human CEMx174 cell line was originally obtained from ATCC (Manassas, VA). Human PBMCs were stimulated for 3 days with $5 \mu \mathrm{g} / \mathrm{ml}$ of phytohemagglutinin (PHA) and 2 U (IL-2) in 10\% FCS RPMI 1640. CD4+ or CD8+ cells were enriched by negative depletion using, respectively, anti-CD8 or anti-CD4 magnetic beads, in combination with anti-CD14 magnetic beads (Dynal, Lake Success, N.Y.) at saturating concentrations. The purity of T-cell subsets was determined for each subset by flow cytometric analysis of cells immunostained with $20 \mu \mathrm{g} / \mathrm{ml}$ anti-CD4 and anti-CD8 monoclonal antibodies (MAbs) detected with FITC-goat anti-mouse IgG. HIV blocking anti-CD4 MAb Leu3A (BD Biosciences, San Jose, CA, USA) was used at $10 \mu \mathrm{g} / \mathrm{ml}$. Soluble CD4-IgG (Progenics) was used at $10 \mu \mathrm{g} / \mathrm{ml}$ during 30 minute preincubations with SIV, just prior to infection of cells.

Flow measurement of CKR expression on normal hPBMCs stimulated with PHA + IL-2 employed anti-CCR5 $\mathrm{mAb} 2 \mathrm{D} 7$ for flow cytometricstainining developed with goat anti-mouse IgG-FITC; anti-CXCR4 mAb 12 G5 was detected by flow cytometetry with goat anti-mouse IgG$\mathrm{PE}$, In these experiments, anti-CD4 mAb was conjugated to either FITC or PE, depending on the CKR mAb being used.

This work was approved by the Institutional Review Board of the Bloomberg School of Public Health, in conformance with the Helsinki Declaration on rights of human subjects.

\section{Virus infection}

SIVmac239 and SIV17E-Fr [28], a kind gift from Dr. Janice Clements, were passaged in human derived CEMx174 cells and purified from culture supernatants by sucrose density gradient centrifugation. The resulting SIV17E-Fr was essentially identical to the starting strain, but the SIV mac239 derived stock virus was found to differ in 15 of 15 clones from the published sequence of SIVmac 239 by three mutations previously associated with CD4 independence. Thus, we refer to this CEMx174 stock strain throughout the text as SIV mac239 ${ }_{\text {CEMx174. }}$

CEMx174 passaged stock viruses were serially passaged in PHA + IL-2 (2 IU) stimulated human PBMCs obtained from normal control donors. Cultures were refed with fresh medium twice weekly, and passaged into 
fresh allogeneic human PBMCs every week. PHA + IL-2 stimulated PBMCs were pretreated with Leu3A (or mock treated) for 30 minutes on ice, and infected with stock SIV $\left(10^{3} \mathrm{TCID}_{50}\right)$, or SIV from various passages (culture supernatant containing $~ 5 \mathrm{ng} / \mathrm{ml} \mathrm{p} 27$ ) for $2 \mathrm{hrs}$ at $37{ }^{\circ} \mathrm{C}$. After infection, the cells were washed free of virus $\mathrm{x} 2$, and plated at $1 \times 10^{6}$ per well in quadruplicate wells of a 48-well culture plate. Supernatants collected on days $0,3,7$ and 10 were assayed for p27 by enzyme immunoassay (EIA, Coulter Immunology). Day 7 and/or day 10 p27 values were used to assess relative rates of growth among control and experimental groups. Groups were statistically compared by two-way Student's T- test.

\section{PCR amplification and sequencing of SIV env sequences from cell culture supernatants}

Genomic DNA was extracted from culture supernatants of SIVmac239 CEMx174 and SIV 17E-Fr infected human hPBMCs with QIA Amp DNA Blood mini Kit (Qiagen), according to the manufacturer's recommendations. Genomic DNA was used for PCR amplification of the entire SIV env gene in a hot start PCR (Taq Master Mix Kit, Qiagen) according to manufacturer's instructions in a standard PCR buffer, and primers at a concentration of $0.5 \mathrm{uM}, \mathrm{P} 1$ (5' GAGCAATCACGAAAGAGAAGAAGAAC3') and P2 (5' CTGTC CCTGAT TGTATTTCTGTCCC-3') for the first round and primers P3 (5' GCTCTAGAATGGGATG TCTTGGGAATCAGCTGC-3') and P4 (5' CGGATCCTCACAAGA GAGTGGCTCAAGCCC-3') for the second round, as previously described [43].

PCR products were run on a $0.7 \%$ low-melt agarose gel, and the $2.6 \mathrm{~kb}$ env product was extracted and cloned into the pCR2.1-TOPO vector (TOPO TA cloning kit, Invitrogen) according to manufacturer's instructions. Fifteen full-length env clones from each stock SIV virus, passages 7 and 24 were randomly selected and sequenced bi-directionally at the Johns Hopkins Sequencing Facility using the M13F and M13R primers with the dye terminator cycle sequencing method and 3730 DNA Analyzer (Applied Biosystems). Sequence analysis was done using SeqMan II, part of Lasergene v 4.03 (DNA Star Inc.). Multiple sequence alignment was done using CLUSTAL $\mathrm{X}$ with weighted residue weight table [44], built into the MegAlign, part of Lasergene version 4.03 (DNA Star Inc.).

\section{Competing interests}

The authors declare that they have no competing interests.

\section{Authors' contributions}

DS conceived the project, participated in the design and interpretation of the experiments, and drafted the manuscript. SI designed experiments, analyzed the genetic data, and participated in drafting and editing the manuscript. Both authors approved the final manuscript.

\section{Acknowledgments}

This work was supported by a CFAR Pilot Grant Developmental Award from CFAR grant \#P30 Al42855 and a grant to DS from the Alternatives Research Development Foundation.

Received: 20 January 2012 Accepted: 25 July 2012

Published: 25 July 2012

\section{References}

1. Reeves JD, Doms RW: Human immunodeficiency virus type 2. J Gen Virol 2002, 83:1253-1265.

2. Wolfe ND, Heneine W, Carr JK, Garcia AD, Shanmugam V, Tamoufe U, Torimiro JN, Prosser AT, Lebreton M, Mpoudi-Ngole E, McCutchan FE, Birx DL, Folks TM, Burke DS, Switzer WM: Emergence of unique primate Tlymphotropic viruses among central African bushmeat hunters. Proc Natl Acad Sci USA 2005, 102:7994-7999.

3. Vodros D, Thorstensson R, Doms RW, Fenyo EM, Reeves JD: Evolution of corecptor use and CD4-independence in evelope clones derived from SIVsm-infected macaques. Virology 2003, 216:17-28.

4. Borsetti A, Parolin C, Ridolfi B, Sernicola L, Geraci A, Ensoli B, Titti F: CD4-independent infection of two CD4(-)/CCR5(-)/CXCR4(+) pre-T-cell lines by human and simian immunodeficiency viruses. J Virol 2000, 74:6689-6694.

5. Roederer M, Mattapallil J: CCR5 and HIV: the less, the better. Blood 2007, 109:854.

6. Pandrea I, Apertrei C, Gordon S, et al: Paucity of CD4 + CCR5+ T cells is a typical feature of natural SIV hosts. Blood 2007, 109:1069-1076.

7. Benhur L, Sharron M, Montaner L, Weissman D, Doms RW: Quantification of CD4, CCR5, and CXCR4 levels on lymphocyte subsets, dendritic cells, and differentially conditioned monocyte-derived macrophages. Proc Natl Acad Sci USA 1999, 96:5215-5220.

8. Lin G, Lee B, Haggarty BS, Doms RW, Hoxie JA: CD4-independent use of Rhesus CCR5 by human immunodeficiency virus Type 2 implicates an electrostatic interaction between the CCR5 $\mathrm{N}$ terminus and the gp120 C4 domain. J Virol 2001, 75:10766-10778.

9. Bhattacharya J, Peters PJ, Clapham PR: CD4-independent infection of HIV and SIV: implications for envelope conformation and cell tropism in vivo. AIDS 2003, 17(Suppl 4):S35-S43.

10. Kuwata T, Byrum R, Whitted S, Gocken R, Buckler-White A, Plishka R, lyengar $R$, Hirsch VM: A rapid progressor-specific variant clone of Simian Immunodeficiency Virus replicates efficiently in vivo only in the absence of immune responses. J Virol 2007, 81:8891-8904.

11. lyengar S, Hildreth JEK, Schwartz DH: Actin-dependent receptor colocalization required for HIV entry into host cells. J Virol 1998, 72:5251-5255.

12. Tardif MR, Tremblay MJ: Regulation of LFA-1 activity through cytoskeleton remodeling and signaling components modulates the efficiency of HIV type-1 entry in activated CD4+ T lymphocytes. J Immunol 2005, 175:926-935.

13. Tardif MR, Tremblay MJ: LFA-1 is a key determinant for preferential infection of memory CD4+ T cells by7 human immunodeficiency virus type 1. J Virol 2005, 79:13714-13724.

14. Chang JT, Palanivel VR, Kinjyo I, Schambach F, Intlekofer AM, et al: Asymmetric lymphocyte division in the initiation of adaptive immune responses. Science 2007, 315:1687-1691.

15. Puffer BA, Pohlmann S, Edinger AL, Carlin D, Sanchez MD, Reitter J, Watry DD, Fox HS, Desrosiers RC, Doms RW: CD4 independence of simian immunodeficiency virus Envs is associated with macrophage tropism, neutralization sensitivity, and attenuated pathogenicity. J Virol 2002, 76:2595-2605.

16. Puffer BA, Altamura LA, Pierson TC, Doms RW: Determinants within gp120 and gp41 contribute to CD4 independence of SIV Envs. Virology 2004, 327:16-25.

17. Overholser ED, Babas T, Zink MC, Barber SA, Clements JE: CD4-independent entry and replication of simian immunodeficiency virus in primary rhesus macaque astrocytes are regulated by the transmembrane protein. J Virol 2005, 79:4944-4951.

18. Gorry PR, Taylor J, Holm GH, Mehle A, Morgan T, Cayabyab M, Farzan M, Wang H, Bell JE, Kunstman K, Moore JP, Wolinsky SM, Gabuzda D: Increased CCR5 affinity and reduced CCR5/CD4 dependence of a neurovirulent 
primary human immunodeficiency virus type 1 isolate. J Virol 2002, 76:6277-6292.

19. Dehghani H, Puffer BA, Doms RW, Hirsch VM: Unique pattern of convergent envelope evolution in simian immunodeficiency virusinfected rapid progressor macaques: association with CD4-independent usage of CCR5. J Virol 2003, 77:6405-6418.

20. Saha K, Zhang J, Gupta A, Dave R, Yimen M, Zerhouni B: Isolation of primary HIV-1 that target CD8+ T lymphocytes using CD8 as a receptor. Nat Med 2001, 7:65-72. Erratum in: Nat Med 2001 7:255.

21. Zerhouni B, Nelson JA, Saha K: Isolation of CD4-independent primary human immunodeficiency virus type 1 isolates that are syncytium inducing and acutely cytopathic for CD8+ lymphocytes. J Virol 2004, 78:1243-1255. Erratum in: J Virol. 78: 6707.

22. Kuwata $T$, Dehghani $H$, Brown CR, Plishka R, Buckler-White A, Igarashi $T$, Mattapallil J, Roederer M, Hirsch VM: Infectious molecular clones from a Simian Immunodeficiency Virus-infected rapid progressor (RP) macaque: evidence of differential selection of RP-specific envelope mutations in vitro and in vivo. J Virol 2005, 80:1463-1475.

23. lyengar S, Schwartz DH, Hildreth JEK: T cell tropic HIV gp120 mediates CD4 and CD8 cell chemotaxis through CXCR4 independent of CD4: Implications for HIV pathogenesis. J Immunol 1999, 162:6263-6267.

24. lyengar S, Schwartz DH, Clements JE, Hildreth JEK: CD4-independent, CCR5-dependent simian immunodeficiency virus infection and chemotaxis of human cells. J Virol 2000, 74:6720-6724.

25. Pohlmann S, Davis C, Meister S, Leslie GJ, Otto C, Reeves JD, Puffer BA, Papkalla A, Krumbiegel M, Marzi A, Lorenz S, Munch J, Doms RW, Kirchhoff F: Amino acid 324 in the simian immunodeficiency virus SIVmac V3 loop can confer CD4 independence and modulate the interaction with CCR5 and alternative coreceptors. J Virol 2004, 78:223-232.

26. Bonavia A, Bullock BT, Gisselman KM, Margulies BJ, Clements JE: A single amino acid change and truncated TM are sufficient for simian immunodeficiency virus to enter cells using CCR5 in a CD4-independent pathway. Virology 2005, 341:12-23.

27. Newstein M, Desrosiers RC: Changes in SIVmac239 gp120 that Compensate for an Inactivating Mutation in the CD4-Binding Domain. Abstract of the $7^{\text {th }}$ Conference on Retroviruses and Opportunistic Infections: 196. January 30February 2, 2000. Abstract \#646.

28. Flaherty MT, Hauer DA, Mankowski JL, Zink MC, Clements JE: Molecular and biological characterization of a neurovirulent molecular clone of simian immunodeficiency virus. J Virol 1997, 71:5790-5798.

29. Demma LJ, Logsdon JM Jr, Vanderford TH, Feinber MB, Staprans SI: SIVsmquasispecies adaptation to a new simian host. PLOS Pathog 2005, 1(1):e3. Epub 2005 Sep 30.

30. Sourisseau M, Sol-Foulon N, Porrot F, Blanchet F, Schwartz O: Inefficient Human Immunodeficiency Virus Replication in Mobile Lymphocytes. J Virol 2007, 81:1000-1012.

31. Orloff SL, Kennedy SM, Belperron AA, Maddon PJ, McDougal S: Two Mechanisms of Soluble CD4 (sCD4)-Mediated Inhibition of Human Immunodeficiency Virus Type 1 (HIV-1) Infectivity and Their Relation to Primary HIV-1 Isolates with Reduced Sensitivity to sCD4. J Virol 1993, 67:1461-1471.

32. Pikora C, Wittish C, Desrosiers RC: Identification of two N-linked glycosylation sites within the core of the simian immunodeficiency virus glycoprotein whose removal enhances sensitivity tosoluble CD4. J Virol 2005, 79:12575-12583.

33. Salzwedel K, Smith ED, Dey B, Berger EA: Sequential CD4-coreceptor interactions in human immunodeficiency virus type 1 Env function: soluble CD4 activates Env for coreceptor-dependent fusion and reveals blocking activities of antibodies against cryptic conserved epitopes on gp120. J Virol 2000, 74:326-333.

34. Haim H, Si Z, Madani N, Wang L, Courter JR, Princiotto A, Kassa A, DeGrace M, McGee-Estrada K, Mefford M, Gabuzda D, Smith AB 3rd, Sodroski J: Soluble CD4 and CD4-mimetic compounds inhibit HIV-1 infection by induction of a short-lived activated state. PLoS Pathog 2009, 4:e1000360. Epub 2009 Apr 3.

35. Crooks ET, Jiang P, Franti M, Wong S, Zwick MB, Hoxie JA, Robinson JE, Moore PL, Binley JM: Relationship of HIV-1 and SIV envelope glycoprotein trimer occupation and neutralization. Virology 2008, 377:364-378.

36. White TA, Bartesaghi A, Borgnia MJ, de la Cruz MJ, Nandwani R, Hoxie JA, Bess JW, Lifson JD, Milne JL, Subramaniam S: Three-dimensional structures of soluble CD4-bound states of trimeric simian immunodeficiency virus envelope glycoproteins determined by using cryo-electron tomography. J Virol 2011, 85:12114-12123.

37. Lauren A, Vincic E, Hoshino H, Thorstensson R, Fenyo EM: CD4-independent use of the CCR5 receptor by sequential primary SIVsm isolates. Retrovirology 2007, 4:50-63.

38. Del Prete GQ, Haggarty B, Leslie GJ, Jordan AP, Romano J, Wang N, Wang J, Holmes MC, Montefiori DC, Hoxie JA: Derivation and Characterization of a Simian Immunodeficiency Virus SIVmac239 Variant with Tropism for CXCR4. J Virol 2009, 83:9911-9922.

39. Bleul CC, Wu L, Hoxie JA, Springer TA, Mackay CR: The HIV coreceptors CXCR4 and CCR5 are differentially expressed and regulated on human T lymphocytes Proc. Natl. Acad. Sci. USA 1997, 94:1925-1930.

40. Zhuang K, Finzi A, Tasca S, Shakirzyanova M, Knight H, Westmoreland S, Sodroski J, Cheng-Mayer C: Adoption of an "open" envelope conformation facilitating CD4 binding and structural remodeling precedes coreceptor switch in R5 SHIV-infected macaques. PLoS One 2011, 6(7):e21350. Epub 2011 Jul 8.

41. Manel N, Hogstad B, Wang Y, Levy DE, Unutmaz D, Littman DR: A cryptic sensor for HIV-1 activates antiviral innate immunity in dendritic cells. Nature 2010, 467:214-217.

42. Laguette N, Sobhian B, Casartelli N, Ringeard M, Chable-Bessia C, Ségéral E, Yatim A, Emiliani S, Schwartz O, Benkirane M: SAMHD1 is the dendritic- and myeloid-cell-specific HIV-1 restriction factor counteracted by Vpx. Nature 2011, 474:654-657.

43. Ryzhova EV, Crino P, Shawver L, Westmoreland SV, Lackner A, Gonzalez-Scarano F: Simian Immunodeficiency Virus encephalitis: analysis of envelope sequences from individual brain multinucleated giant cells and tissue samples. Virology 2002, 297:57-67.

44. Thompson JD, Higgins DG, Gibson TJ: CLUSTAL W: improving the sensitivity of progressive multiple sequence alignment through sequence weighting, position-specific gap penalties and weight matrix choice. Nucleic Acids Res 1994, 22:4673-4680.

doi:10.1186/1742-4690-9-61

Cite this article as: lyengar and Schwartz: Acquisition of CD4-

Dependence by CD4-Independent SIV Passaged in Human Peripheral Blood Mononuclear Cells. Retrovirology 2012 9:61.

\section{Submit your next manuscript to BioMed Central and take full advantage of:}

- Convenient online submission

- Thorough peer review

- No space constraints or color figure charges

- Immediate publication on acceptance

- Inclusion in PubMed, CAS, Scopus and Google Scholar

- Research which is freely available for redistribution 